\title{
VERTICAL STRUCTURE OF NGC 4631
}

\author{
Hong Bae Ann ${ }^{1}$, Mira SeO ${ }^{1}$, And Su-Ja BaeK ${ }^{2}$ \\ ${ }^{1}$ Division of Science Education, Pusan National University, Busan 609-735, Korea \\ E-mail : hbann@pusan.ac.krandmrseo@pusan.ac.kr \\ ${ }^{2}$ Ulsan High School, Ulsan 681-222, Korea \\ E-mail : whitesuji@hanmail.net \\ (Received January 07, 2011; Revised January 17, 2011; Accepted January 18, 2011)
}

\begin{abstract}
We present a deep CCD imaging in $B$ and $V$ bands which allows us to analyze the vertical structure of NGC 4631. We derive the scale heights of the thin and thick disks at a variety of positions along the major axis of the disk. The scale heights of the thin disk are nearly constant while those of the thick disk tend to increase with increasing galactocentric distance. The mean scale heights of the thin disk derived from $B$ and $V$ images are similar to each other $(\sim 450 \mathrm{pc})$. Instead, those of the thick disk show a strong east-west asymmetry which is caused by the diffuse stellar emission that is most prominent in the north west regions above the disk plane. The ratio of scale heights $\left(z_{\text {thick }} / z_{\text {thin }}\right)$ is about 2.5 in the east side of the disk. However, this ratio is greater than 4 for the thick disk above the disk plane in the west side of the galaxy.
\end{abstract}

Key words : galaxies: individual — NGC 4631 — galaxies: disk — galaxies: scale height

\section{INTRODUCTION}

The disk of a galaxy had been thought to be very thin until the discovery of thick disk in some edge-on galaxies (Burstein 1979; Tsikoudi 1979) and in the Milky Way (Gilmore \& Reid 1983). Since most of baryons reside in the galaxy disks, understanding the structure of the galactic disks is crucial in the theory of galaxy formation. A thin disk is believed to be formed by quiescent dissipation of baryons after virialization of the dark halo (e.g., Fall \& Efstathiou (1980)). However, formation of thick disks is not as clear as that of a thin disk.

Many scenarios have been proposed to explain the origin of thick disks, e.g., Yoachim \& Dalcanton (2008), and references therein. The first family of scenarios assumes that the thick disk is a separate component formed during the initial rapid proto-galactic collapse (Jones \& Wyse 1983; Gilmore 1984; Burkert et al. 1992; Kroupa 2002; Fuhmann 2004) or during a chaotic period of hierarchical clustering at high redshift (Brook et al. 2004). The second family of scenarios assumes that the thick disk is formed from tidally stripped debris of accreted satellite galaxies (Gilmore, Wyse \& Norris 2003; Abadi et al. 2003; Martin et al. 2004; Navarro, Helmi \& Freeman 2004; Helmi et al. 2006; Kazantzidis et al. 2008; Read et al. 2008). The third family assumes that it is an extension of the thin disk heated by a variety of ways among which heating by satellite accretion (Carney et al. 1989; Quinn et al. 1993; Robin et al. 1996; Walker, Mihos \& Hernquist

Corresponding Author: H. B. Ann
1996; Velazquez \& White 1999; Bekki \& Chiba 2001; Vilalobos \& Helmi 2008) is thought to be most relevant to the thick disks of galaxies with close companions. Freeman \& Bland-Hawthorn (2002) assume that the satellite accretion which led to the formation of the thick disk occurs soon after the disk began to settle and the morphology of the thin disk depends on the numbers of satellite galaxies to be accreted.

The vertical structure of disk galaxies can be understood by examining the surface brightness profiles perpendicular to the plane of the disk. However, it is difficult to analyze the perpendicular profiles because the disk at high $z$ is generally very faint. The first identification of a thick disk in external galaxies was made by Burstein (1979) from a detailed analysis of the perpendicular profiles of a number of edgeon S0 galaxies. The exponential distribution of the perpendicular profiles $\left(\exp \left(-z / z_{h}\right)\right)$ was reported by Burstein (1979) and Tsikoudi (1979) but van der Kruit \& Searle (1981a,b) suggested a hyperbolic function $\left(\operatorname{sech}^{2}\left(z / z_{0}\right)\right)$ for the perpendicular profiles which is expected from the isothermal distribution of stars. Since the above two functions show the same behavior at high $z$ and the exponential nature of the perpendicular profiles was observed for a large number of spiral galaxies (e.g., de Grijs \& Peletier 1997; Pohlen et al. $2000)$, the exponential function is still widely used to represent the vertical luminosity distribution of spiral galaxies.

NGC 4631 is a nearby edge-on spiral galaxy of Hubble type $\mathrm{SB}(\mathrm{s}) \mathrm{d}$. Its distance determined by the tip of the red giant branch (TRGB) method (Lee, Freedman 
\& Madore 1993) is 7.6 Mpc (Seth et al. 2005a). It is an ideal target to study the thick disk which is assumed to be produced by a heating mechanism associated with the accretion of satellite galaxies after the disk was formed. The reason why we expect satellite accretion to be the main cause of disk heating is that NGC 4631 is located in a group environment where more than a dozen galaxies reside (Giuricin et al. 2000). Moreover, a recent wide field imaging survey of Kim et al. (2011) shows that there are plenty of dwarf satellite galaxies around NGC 4631. Since most of the satellite galaxies found by Kim et al. (2011) are thought to be located within the virial radius of NGC 4631, we expect strong on-going tidal and hydrodynamical interactions between NGC 4631 and its satellite galaxies. Among others, the distorted morphology of a dwarf elliptical galaxy NGC 4627 and the HI bridge between NGC 4631 and NGC 4656 (Roberts 1968) are two typical examples of strong interactions between NGC 4631 and its satellite galaxies. NGC 4627 and NGC 4656 are located at 2.6 arcmin to the north-west and 32 arcmin to the south-east. At the distance of 7.6 Mpc for NGC 4631, NGC 4627 and NGC 4656 are separated by about 6 $\mathrm{kpc}$ and $71 \mathrm{kpc}$, respectively, from NGC 4631.

The presence of extraplanar components in NGC 4631 is reported by a variety of observations, from Xrays to radio wavebands (Fabbiano \& Trinchieri 1987; Hummel et al. 1991; Rand 1994; Wang et al. 1995; Vogler \& Pietsch 1996; Neininger \& Dumke 1999; Martin \& Kern 2001; Wang et al. 2001; Bendo et al. 2006) including the recent radio observation of molecular out flow (Irwin et al. 2011). In particular, Martin \& Kern (2001) observed diffuse ionized gas far from the disk using $\mathrm{H}_{\alpha}$ imaging. The extraplanar gas or dust out to $z>1 \mathrm{kpc}$ and diffuse light around the disk affect the disk scale height substantially. Hummel \& Dettmar (1990) showed that the scale height at $r=3^{\prime}$ in the west side of the disk is about 2.5 times larger than that of the east side. Seth et al. (2005a,b) resolved the diffuse stellar emission into individual stars using $H S T / A C S$ high resolution images. They showed that the scale height of red giant branch (RGB) stars is about two times larger than that of the main sequence stars.

The purpose of the present work is to understand the vertical structure of NGC 4631 which seems to be much affected by neighboring galaxies. Since diffuse stellar emission around the galaxy is extremely faint, we conducted deep $B$ and $V$-band CCD imaging from which we extract perpendicular profiles at various radii along the major axis of NGC 4631. In Section 2, we describe our observations and the basic procedures for the surface photometry of galaxies. We present results of the profile analysis in Section 3. Discussion and Summary are provided in the last section.

\section{OBSERVATIONS AND DATA REDUC- TION}

The observations were carried out using the $f 7.5$ $1 \mathrm{~m}$ Lemmonsan telescope equipped with FLI Microline CCD which uses a Kodak full-frame KAF-4301E chip. The CCD has $2084 \times 2084$ pixels. The pixel size is $24 \mu \mathrm{m}$, which corresponds to $\sim 0.64^{\prime \prime}$. It has a readout noise of $15 e$ and a cooling temperature of $\sim 50^{\circ}$ below ambient. The field of view is $22.5^{\prime} \times 22.5^{\prime}$. The observations were carried out remotely at Daeduck for 7 nights from March 202004 to April 202004 since the Lemmonsan Observatory is located at a site in Arizona. We took $55 \mathrm{~B}$-band and $66 \mathrm{~V}$-band images with exposure times of $900 \mathrm{~s}$ and $600 \mathrm{~s}$, respectively. The total exposure times are 13.75 hours and 11 hours for $B$ and $V$-band, respectively.

We followed the basic procedures for CCD reductions using IRAF including bias and dark subtraction and flatfielding. We applied a median combine with a 3 sigma clipping to obtain the deep $B$ and $V$-band images shown in Fig. 1 and Fig. 2. Fig. 1 is presented to display the clumpy structures revealed by the bright substructures while Fig. 2 is presented to display the fainter parts of the galaxy, especially the diffuse stellar emission above the middle plane of the disk. We can see a non-uniform distribution of diffuse stellar emission which is most prominent in the north west regions.

We conducted a detailed surface photometry from the combined $B$ and $V$ images using SPIRAL, a software developed at the Kiso Observatory for the surface photometry of galaxies (Ichikawa et al. 1987). The first step of surface photometry of galaxies is to obtain the relative intensity distribution $I_{r e l}(x, y)$ defined as

$$
I_{r e l}(x, y)=\frac{I_{G+S}(x, y)-I_{S}(x, y)}{I_{S}(x, y)}
$$

where $I_{G+S}(x, y)$ and $I_{S}(x, y)$ are the galaxy intensity distributions which include the light from the sky background and the sky background itself, respectively.

Since the surface brightness of a galaxy is fainter than that of the sky background except in the central part, a small error in the determination of $I_{S}(x, y)$ affects the surface brightness profile significantly. In SPIRAL, the intensity of the local sky background, $I_{S}(x, y)$ is determined by fitting two-dimensional polynomials to the regions around the target galaxy. In most cases, a simple polynomial of the form $I_{S}(x, y)=$ $a_{0}+a_{1} x+a_{2} y$, is sufficient to derive a reasonable $I_{S}(x, y)$. However, for our $B$ and $V$ images of NGC 4631 , an accurate determination of the local sky background, $I_{S}(x, y)$, is very difficult due to the large angular size of NGC 4631 which is comparable to the field of view of the CCD. Moreover scattered light from the telescope optics makes some regions of the CCD too bright. We carefully selected the regions for fitting the polynomials, and examined the residuals after subtracting the fitted sky to determine which order 


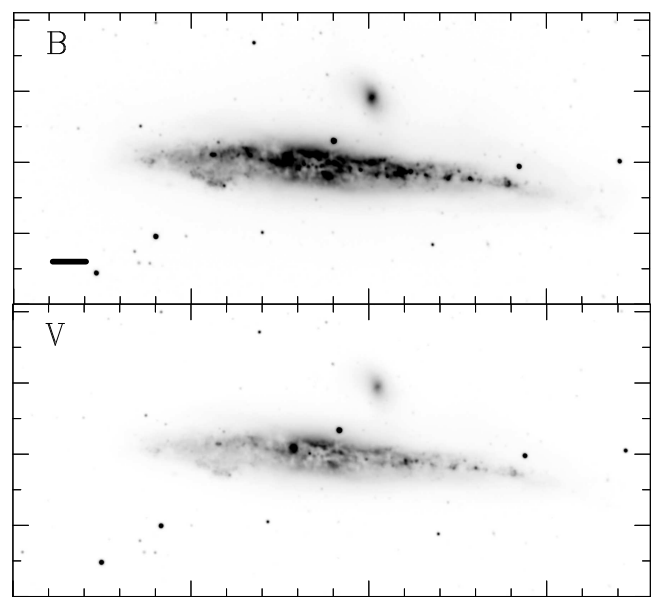

Fig. 1. $-B$ and $V$ images of NGC 4631 . The small galaxy to the north-west of NGC 4631 is NGC 4627. The horizontal scale bar in the lower left corner of the upper panel represents 1 arcmin. North is top, east is to the left.

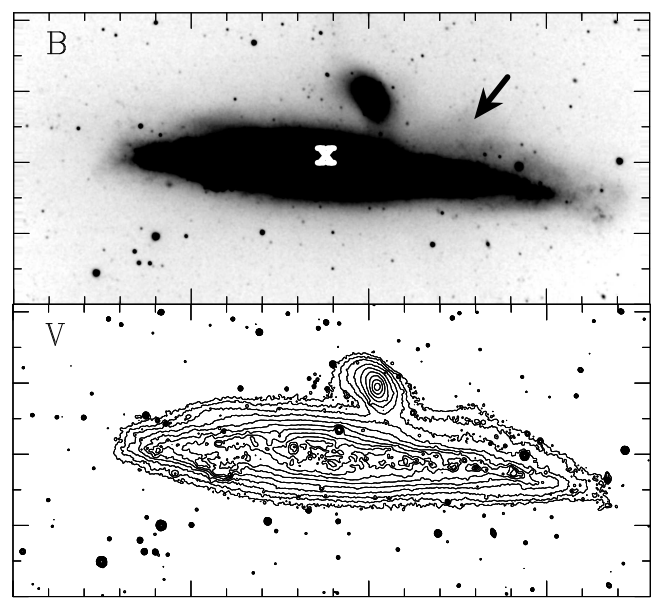

Fig. 2.- Morphology of NGC 4631 shown in a $B$-band image and in a $V$-band isophotal map. The center of the galaxy and the region of the most prominent diffuse stellar emission are indicated respectively by $\mathrm{x}$ and by the arrow in the upper panel. The faintest contour represents a surface brightness of $4.5 \mathrm{mag}$ below the sky brightness and the contour interval is $0.5 \mathrm{mag}$.

gives the most uniform residuals around the galaxy. We found that a third order polynomial in $x$ and $y$, i.e.,

$$
\begin{array}{r}
I_{S}(x, y)=a_{0}+a_{1} x+a_{2} y+a_{3} x^{2}+a_{4} x y+a_{5} y^{2} \\
+a_{6} x^{3}+a_{7} x^{2} y+a_{8} x y^{2}+a_{9} y^{3},
\end{array}
$$

is best suited for the determination of the local sky background, $I_{S}(x, y)$ of NGC 4631 . Since the raw relative intensity distribution, $I_{r e l}(x, y)$, is very noisy, especially in the faint outer parts of the galaxy, we applied a Gaussian beam smoothing after removing bright background stars from the images.

In order to derive the perpendicular profiles from the $B$ and $V$ images, we first rotated the smoothed images to align the major axis of the galaxy parallel to the $\mathrm{x}$-axis (assuming a position angle of $85^{\circ}$ ), and then we extracted profiles along the y-axis with a sampling pitch of $3^{\prime \prime}$ at 10 radii, separated by $\sim 72^{\prime \prime}$, along the major axis. We extracted two perpendicular profiles at a given location, one is oriented towards the north (hereafter $\mathrm{PP}_{N}$ ), the other to the south (hereafter $\left.\mathrm{PP}_{S}\right)$. Thus, the total number of perpendicular profiles to be analyzed for the vertical structure of NGC 4631 is 20 . We used an aperture size that increased with height starting at $4^{\prime \prime}$ to obtain high $\mathrm{S} / \mathrm{N}$ at large distances from the plane. We show the $B$-band and $V$-band perpendicular profiles in Fig. 3 and Fig. 4, respectively. These profiles show the distribution of the surface brightness relative to that of the sky, the latter calibrated by the standard stars observed during the observing runs.

These perpendicular profiles extracted at radii separated evenly along the major axis of the disk are thought to represent the typical luminosity distribution perpendicular to the middle plane of the disk. However, as shown in Fig. 3 and Fig. 4, some parts of the profiles are heavily affected by the foreground/background stars and galaxies so that some of the profiles are difficult to analyze in detail. Thus, we extracted additional PP at radii where the contamination by foreground/background sources is minimal.

\section{RESULTS}

\subsection{Morphology}

As shown in Fig. 1, the morphology of NGC 4631 is very complex. The central part of the galaxy is highly obscured by dust, which make it difficult to determine its center. There are a number of substructures in the disk of NGC 4631; most of them are thought to be associated with young stellar populations which show strong $\mathrm{H}_{\alpha}$ emissions (Crillon \& Monnet 1969; Hummel \& Dettmar 1990; Rand et al. 1992). The blue color $(B-V \approx 0.4)$, the dusty morphology of the central regions of the galaxy and the chains of blue clumps along the major axis of the disk suggest active star formation in NGC 4631 (Hummel \& Dettmar 1990; Rand et al. 1992). There is a strong asymmetry in the luminosity distribution. The major axis in the east side of the galaxy is $\sim 0.6^{\prime}$ shorter than that in the west side. On either side of the disk, the position angle of the major axis varies in the outer part, It increases with radius in the east side and decreases in the west side, displaying a U-shaped warp. Hummel \& Dettmar (1990) reported that the optical disk bends to the south in the western part only but their $B$-band isophotal map shows an isophotal twist in the eastern part too, although too small to be easily recognized. However, considering that most optical warps have small warp amplitudes, $\alpha \approx 3.4^{\circ}$ and $\alpha \approx 3.1^{\circ}$ for S-shaped warps and U-shaped warps, respectively (Ann \& Park 2006), NGC 4631 seems to have a typical U-shaped warp. The 


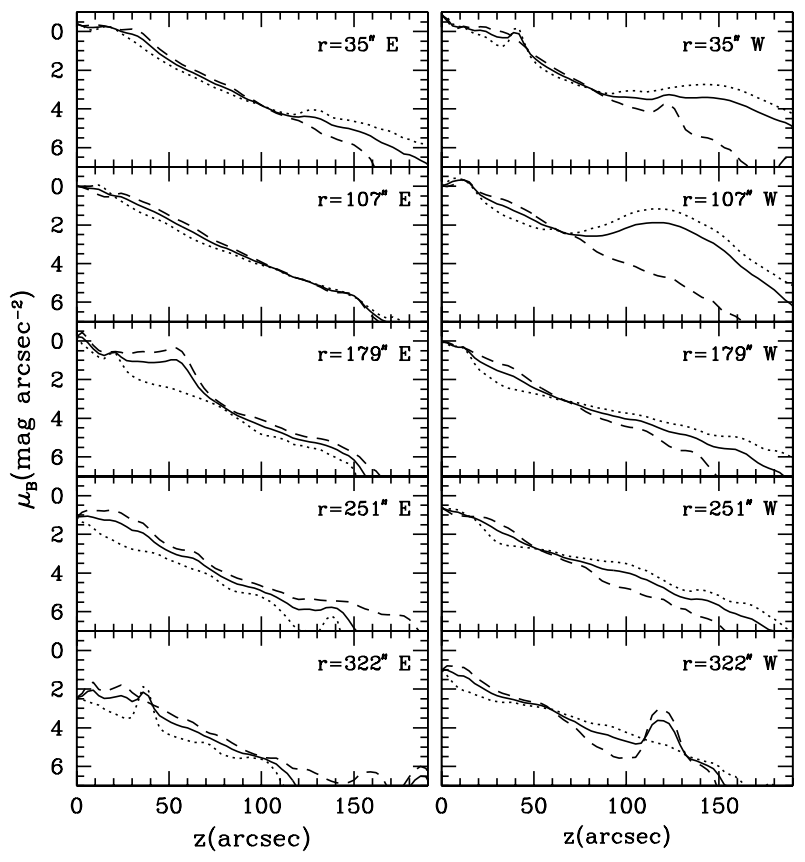

Fig. 3.- $-B$-band surface brightness profiles perpendicular to the major axis of NGC 4631. The dotted lines represent profiles above the disk plane $\left(\mathrm{PP}_{N}\right)$ while the dashed lines designate those below the disk plane $\left(\mathrm{PP}_{S}\right)$. The solid lines represent the averaged profiles. The label in the upper right corner of each panel shows the radius at which the profiles are extracted. The surface brightness is relative to the surface brightness of the sky background, $\mu_{s k y}(B)=21.04$.

slightly bent shape of the NGC 4631 disk was reported by Rand et al. (1992) from $\mathrm{H}_{\alpha}$ imaging and by Weliachew et al. (1978) from HI observations.

The diffuse stellar emission around the galaxy (see Fig. 2) is very complex, showing large east-west and north-south asymmetries. The north-south asymmetry is more pronounced than the east-west asymmetry, and also observed in the HI distribution (Weliachew et al. 1978). The most pronounced feature is the extended region to the west of NGC 4267 in the northwest side of the galaxy, indicated by the arrow in the top panel of Fig. 2. There is less stellar emission below the disk plane. A similar asymmetric diffuse stellar emission was reported for the integral-sign galaxy PGC 20348 (Ann 2007). The origin of the diffuse stellar emission is not clear; it could be caused by interactions with neighboring galaxies such as NGC 4656 and NGC 4627 (Combes 1978; Hummel \& Dettmar 1990). Despite the small separation between NGC 4631 and NGC 4627, it is unlikely that NGC 4627 could detach some stars from the disk of NGC 4631 since the mass of NGC 4627, $\sim 3 \times 10^{7} M_{\odot}$ (Rand 1994) seems to be too small to disturb NGC 4631 significantly. Rather, NGC 4656 which has comparable mass, $\sim 1.5 \times 10^{10} M_{\odot}$ (Roberts 1968 ) could play the role during close encounters. One thing worth noting is that if the diffuse strip from the

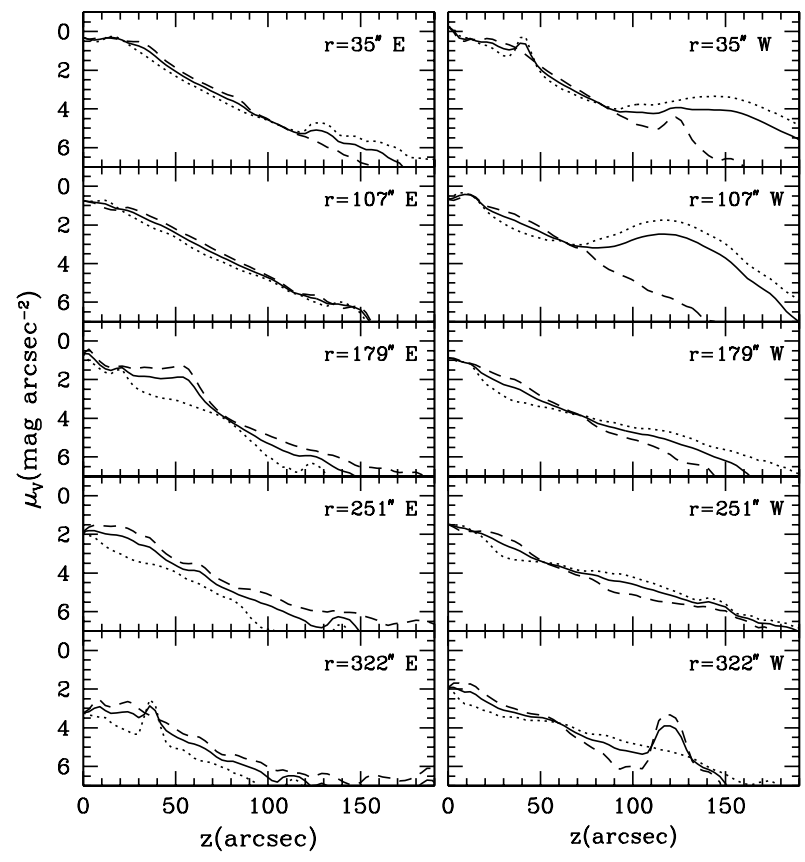

Fig. 4.- $V$-band surface brightness profiles perpendicular to the major axis of NGC 4631. The meanings of the symbols are the same as those in Fig. 3. The surface brightness is relative to the surface brightness of the sky background, $\mu_{\text {sky }}(V)=19.96$.

top in the middle of the image (Fig. 2) that is connected to the north east part of NGC 4627 is a real feature, it can be explained by the model of Combes (1978) which assumes close encounters with NGC 4656 in a parabolic orbit.

\subsection{Major and Minor Axes Profiles}

Fig. 5 shows the surface brightness profiles along the major axis and minor axis of NGC 4631 in $B$ and $V$ bands. The major-axis profiles show very irregular luminosity distributions in the inner part of the galaxy $\left(r<120^{\prime \prime}\right)$ with relatively smooth distribution in the outer parts. The major-axis profile in the east side of the disk is systematically fainter than that in the west side of the disk at $r>220^{\prime \prime}$. This asymmetry seems to be caused by the asymmetric spiral arms which are much longer and brighter in the west side. The bright peaks at $r \approx 150^{\prime \prime}, 240^{\prime \prime}, 280^{\prime \prime}, 340^{\prime \prime}$ are due to the bright substructures which are thought to be HII region complexes. There is not much difference in the luminosity profiles between the $B$-band and $V$-band,

The minor-axis profiles shown in Fig. 5 are nearly straight except for the central regions where the profile shapes are heavily affected by dust. This means that the minor axis profiles are well represented by an exponential function. However, there are at least three components that contribute to the minor-axis profiles. The first and most significant component is the thin disk which is delineated by a rather steep gradient in- 


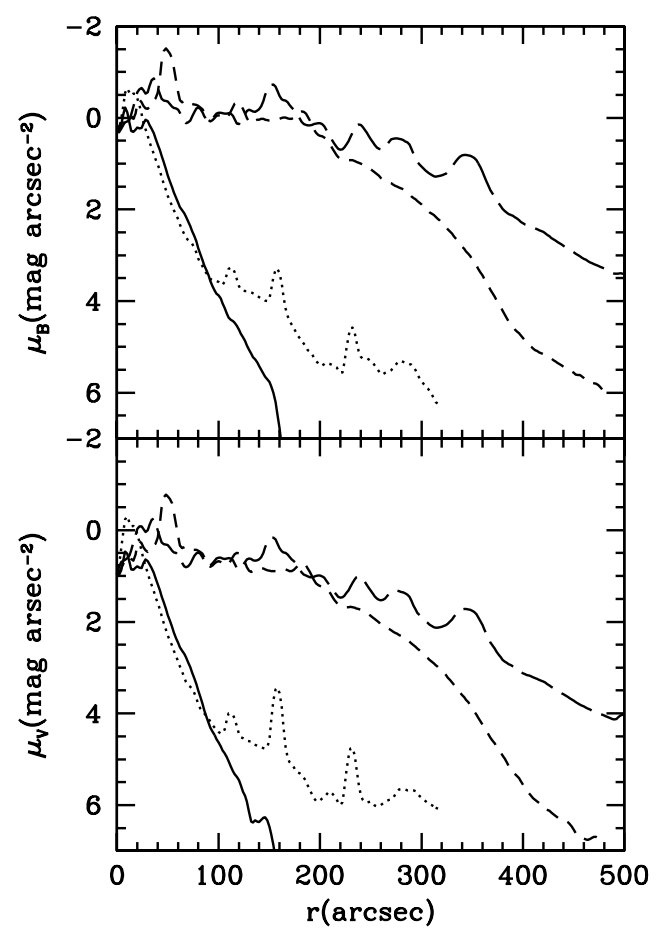

Fig. 5. - Major-axis and monir-axis profiles of NGC 4631. $B$ and $V$ bands are shown in top and bottom panels, respectively. The short dashed lines and long dashed lines indicate the major-axis profiles in the east side and west side of the disk, respectively. The dotted lines and the solid lines represent the minor-axis profiles in the above and below the disk plane.

side $r<50^{\prime \prime}$. The second component is the thick disk which dominates the minor-axis profiles in the outer regions, $r>100^{\prime \prime}$ where the minor-axis profiles show somewhat less steep gradient. The third contribution to the minor-axis profile is the bar component which makes a slight change in the slope of the profile at $r \approx 60^{\prime \prime}$. The thick disk is very pronounced in the northern part of the disk where diffuse stellar emission is more prominent. The minor axis profiles seem little affected by the luminosity from the bulge of NGC4631 perhaps because its bulge is negligibly small.

\subsection{Perpendicular Profiles}

The PP shown in Fig. 3 and Fig. 4 show the typical shape of the luminosity distribution perpendicular to the disk major axis which is characterized by an exponential decline of luminosity. The distinction between the thin disk and the thick disk is not as apparent as that in the minor-axis profiles. However, we can identify the thin and thick disks by the different slopes, steep slopes for the thin disk at $z<50^{\prime \prime}$ and less steep slopes for the thick disk at $z>70^{\prime \prime}$ for most of the profiles.

In the east side of the galaxy, the luminosity distributions as a function of the distance perpendicular to the major axis $(z)$ are very symmetric about the disk major axis. In the west side of the galaxy, however, the luminosity distributions as a function of $z$ are symmetric at $z<70^{\prime \prime}$ and asymmetric thereafter. The reason for the large asymmetries in the PP extracted at $r=35^{\prime \prime} \mathrm{W}$ and $r=107^{\prime \prime} \mathrm{W}$ is different from the reason for the large asymmetries in PP extracted at $r \geq 179^{\prime \prime} \mathrm{W}$. The former is caused by the luminosity of a nearby dwarf galaxy NGC 4627, while the latter is due to the diffuse stellar emission above the middle plane of the disk (see Fig.2). The plateau in $\mathrm{PP}_{N}$ at $r=179^{\prime \prime} \mathrm{E}$ and $z \approx 40^{\prime \prime}$, and the bumps at $r=322^{\prime \prime}$ E and $z \approx 35^{\prime \prime}, r=35^{\prime \prime} \mathrm{W}$ and $z \approx 40^{\prime \prime}$ and $r=322^{\prime \prime}$ $\mathrm{W}$ and $z \approx 120^{\prime \prime}$ are caused by foreground stars.

It is of interest to see that the PP are very symmetric about the disk major axis at small height, $z<70^{\prime \prime}$, even for the PP measured in the west side of the disk where large amounts of disk material are supposed to be detached by strong encounters with neighboring galaxies (Combes 1978). Even though tidal forces due to neighbor galaxies are thought to be strong enough to make the peculiar morphology of NGC 4631, characterized by large asymmetric spiral arms and warps, the vertical structure of the inner disk seems to be very stable against the external forces that may distort the radial structure of the galaxy.

\subsection{Vertical Structures}

The luminosity profile perpendicular to the major axis of the disk consists of the luminosities from the thin and thick disk components. However, the luminosity profile at small $z$ is dominated by the thin disk while that at large $z$ is dominated by the thick disk. As a first approximation, we determined the disk scale heights of NGC 4631 by fitting a single exponential function,

$$
I(z)=I_{o}(r) \exp \left(-z / z_{h}\right)
$$

to the $\mathrm{PP}_{S}$ and $\mathrm{PP}_{N}$ in Fig. 3 and Fig. 4 at $z>70^{\prime \prime}$. In the above equation $I_{o}(r)$ and $z_{h}$ are the central intensity at $r$ along the major axis of the disk and the disk scale height, respectively. The scale heights derived in this way seem to represent the scale heights of the thick disk component because the thick disk dominates the luminosity profile at large $z$.

Fig. 6 shows the variation of the thick disk scale height $\left(z_{h}\right)$ of NGC 4631 as a function of radius along the major axis. The scale heights derived from the PP in the eastern part of the galaxy are represented by open symbols while those from the PP in the western part of the galaxy are plotted as filled symbols. The disk scale heights are mean values from the three profiles separated by $10^{\prime \prime}$ which is supposed to be the maximum uncertainty of the position of the galaxy center along the major axis of the galaxy. The error bars are the standard deviations of the mean disk scale heights. The scale heights derived from the $B$ and $V$ perpendicular profiles below the disk plane $\left(\mathrm{PP}_{S, B}\right.$ and $\left.\mathrm{PP}_{S, V}\right)$ 


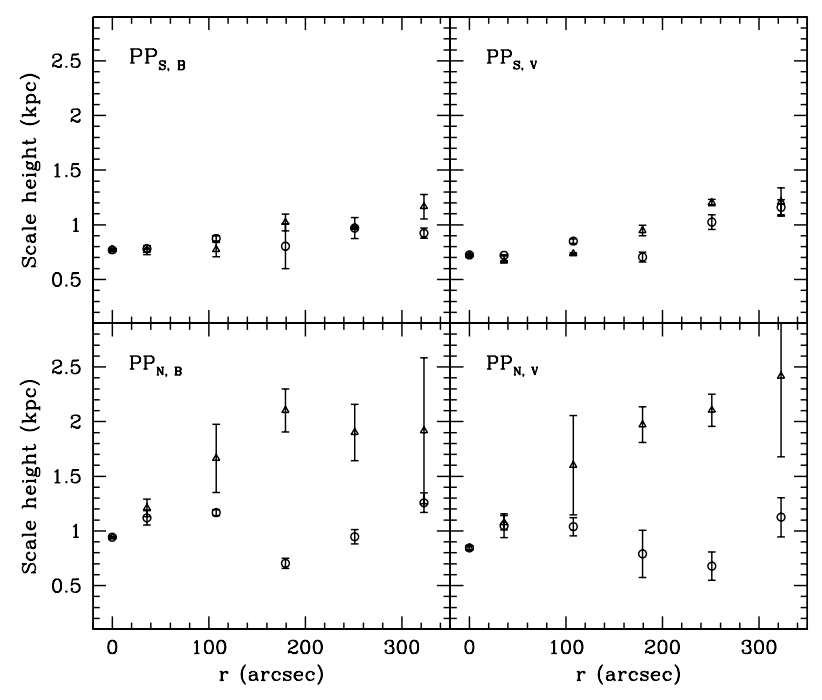

Fig. 6. - Scale heights of the thick disk as a function of radius along the major axis of the disk. Open circles indicate the scale heights derived from the surface brightness profiles extracted from the eastern part of the disk while open triangles represent the scale heights of the western part of the disk. The error bars are the standard deviations of the scale heights derived from the three profiles separated by $10^{\prime \prime}$. The labels in the upper left corner of each panel indicate profiles used. $\mathrm{PP}_{S, B}$ and $\mathrm{PP}_{N, B}$ indicate the perpendicular profiles orientated to the south and north, respectively, in $B$-bands whereas $\mathrm{PP}_{S, V}$ and $\mathrm{PP}_{N, V}$ represent those in $V$-band.

seem to increase with increasing radius along the disk major axis on both sides of the disk. Similar increase of disk scale heights were observed for a sample of 48 edgeon galaxies (de Grijs \& Peletier 1997) which employed nearly the same method as that used here. There is a large discrepancy between the scale heights of the east side and those of the west side for the $B$ and $V$ perpendicular profiles above the disk plane $\left(\mathrm{PP}_{N, B}\right.$ and $\left.\mathrm{PP}_{N, V}\right)$. The scale heights of the west side of the disk are about two times larger than those of the east side at $r>70^{\prime \prime}$. There is no difference between the scale heights derived from different passbands. The large east-west asymmetry for $\mathrm{PP}_{N, B}$ and $\mathrm{PP}_{N, V}$ is due to diffuse stellar emission which is most prominent in the north west regions above the disk plane.

Since the luminosity of the thin disk contributes to the profiles where the thick disk dominates, it is better to take into account the luminosity of the thin disk in the derivation of the scale heights of the thick disk. Thus, we applied an iterative profile decomposition technique (Kormendy 1977) to the $16 \mathrm{PP}$ which are not much affected by the foreground stars and back- ground galaxies. The selected PP include the minoraxis profiles (Fig. 5) and the perpendicular profiles extracted at $r=107^{\prime \prime} \mathrm{E}$ and $r=179^{\prime \prime} \mathrm{W}$ shown in Fig. 3 and Fig.4. In the profile decomposition, we considered the thin and thick disks only and neglected other components such as bulge and bar because the contributions of these components are negligibly small even in the minor axis profiles. We assumed exponential functions, $I_{\text {thin }}(z) \propto \exp \left(-z / z_{\text {thin }}\right)$ and $I_{\text {thick }}(z) \propto \exp \left(-z / z_{\text {thick }}\right)$, respectively for the thin disk and the thick disk where $z_{\text {thin }}$ and $z_{\text {thick }}$ are scale heights. Iterative fitting technique seems to be effective because it determines only two parameters at a time by least-square fittings at the regions where the assumed component dominates. The fitting regions of each component are different from profile to profile but in most cases the thin disk component is fitted at $z=10^{\prime \prime} \sim 20^{\prime \prime}$ while the thick disk component is fitted at $z=80^{\prime \prime} \sim 120^{\prime \prime}$, respectively.

Fig. 7 shows the scale heights determined by the profile decomposition of the $16 \mathrm{PP}$ which are presented in Fig. 8 and Fig. 9 in the Appendix. The scale heights of the thin disk, plotted as open circles for $\mathrm{PP}_{N}$ and filled circles for $\mathrm{PP}_{S}$, show no dependence on the galactocentric distance, whereas the scale heights of the thick disk, plotted as open triangles for $\mathrm{PP}_{N}$ and filled triangles for $\mathrm{PP}_{S}$ show significant variations in the west side of the disk for $\mathrm{PP}_{N}$. The mean scale heights of the thin disk are $400 \pm 140$ pc and $520 \pm 150$ pc, respectively for $B$-band and $V$-band, whereas those of the thick disk derived from the $\mathrm{PP}_{S}$ which show nearly constant scale heights are $1330 \pm 250 \mathrm{pc}$ and $1250 \pm 280$ for $B$-band and $V$-band, respectively. Thus, the ratio of the scale heights, $z_{\text {thick }} / z_{\text {thin }}$ is $\sim 3.3$ for $B$-band and $\sim 2.5$ for $V$-band. The large scale heights for the thick disk in the west side of the disk are due to diffuse stellar emission which is most prominent there.

\section{DISCUSSION AND SUMMARY}

We have derived the scale heights of the thin and thick disks of NGC 4631 using extremely deep CCD images in $B$ and $V$ bands. We selected several positions along the disk major axis which are less affected by foreground and background sources to measure representative perpendicular profiles of NGC 4631. The mean scale heights of the thin disk are $\sim 450 \mathrm{pc}$ for $B$-band and $V$-band. The mean scale heights of the thick disk derived by fitting a single exponential function to the $\mathrm{PP}_{S}$ at $z>70^{\prime \prime}$ are $\sim 900$ pc for $B$-band and $V$-band. Thus the ratio of thick disk scale height to thin disk scale height, $z_{h} / z_{\text {thin }}$ is about 2 which is similar to that determined by Dalcanton \& Bernstein (2002) and Yoachim \& Dalcanton (2006). Since $\mathrm{PP}_{S}$ are less affected by diffuse stellar emission which makes the slope of the profile flatter, it would be the lower limit for $z_{\text {thick }} / z_{\text {thin }}$.

The scale heights of the thick disk derived by an iterative profile decomposition technique from the $\mathrm{PP}_{S}$ are 


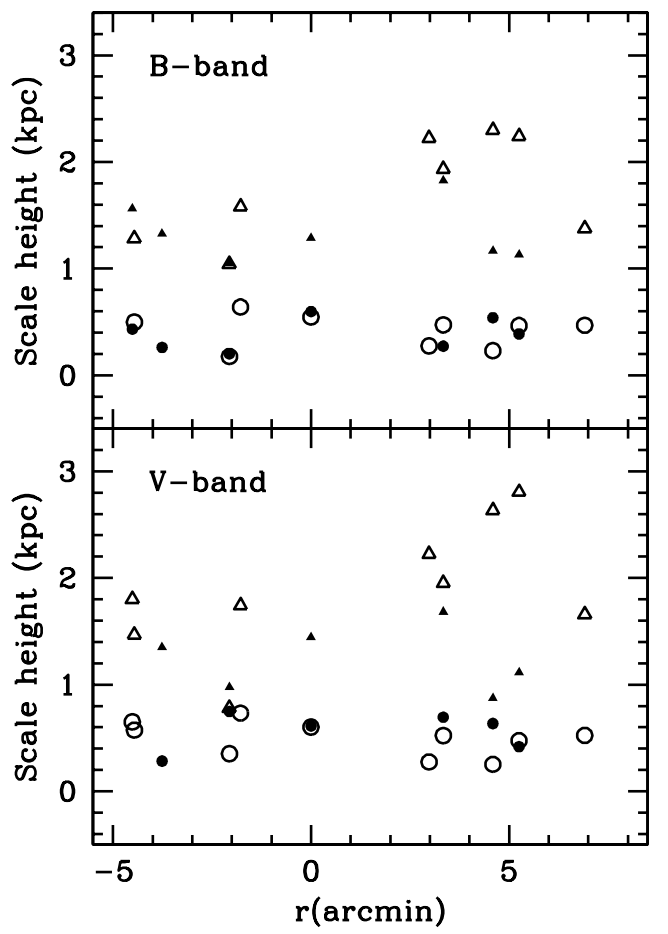

Fig. 7. - Scale heights of the thick disk as a function of the galactocentric distance. The circles and triangles indicate the scale heights of the thin disk and those of the thick disk, respectively. We designate the scale heights from the $\mathrm{PP}_{N}$ as open symbols while those from the $\mathrm{PP}_{S}$ as filled symbols. The minus values of $r$ in the abscissa represent the radii along the disk major axis in the east side of the disk, while the plus values indicate those in the west side of the disk.

$\sim 1300 \mathrm{pc}$ and $\sim 1250 \mathrm{pc}$, respectively for $B$-band and $V$-band. These values of the thick disk scale heights are about two times larger than those of RGB stars in the region above the disk plane of NGC 4631 (Seth et al. 2005b). However, their scale height of $z_{0} \approx 1400$ pc for isothermal sech ${ }^{2}$ profile is comparable to those derived by fitting a single exponential function to $\mathrm{PP}_{S}$ since $z_{0}$ corresponds to $z_{h}=700 \mathrm{pc}$. The scale height ratio of $z_{\text {thick }} / z_{\text {thin }} \approx 3$ is similar to that of Pohlen et al. (2004) who derived scale heights by iterative profile decomposition.

The thin disk of NGC 4631 has nearly constant scale heights along the disk major axis, while the thick disk has scale heights that increase with increasing galactocentric distance. The constancy of disk scale heights in spiral galaxies was found for a sample of edge-on spiral galaxies (van der Kruit \& Searle 1981a,b). van der Kruit \& Searle (1982) suggested that the constant scale heights are the results of secular accelerations of disk stars by massive molecular clouds (Spitzer \& Schwarzschild 1951) of which the number density is proportional to the star formation rate. However, the constancy of scale heights of the thin disk of NGC 4631 is of special interest because NGC 4631 is thought to suffer strong tidal interactions that might detach some of the stellar disk of NGC 4631. This either means that the thin disk of NGC 4631 is very stable against the tidal forces that might detach some stars from the disk, or that the effect of tidal forces on the thin disk is independent of the galactocentric distance.

The scale heights of the thick disk from the perpendicular profiles below the disk middle plane $\left(\mathrm{PP}_{S}\right)$ show little variation along the major axis, whereas those of the thick disk from the perpendicular profiles above the disk middle plane $\left(\mathrm{PP}_{N}\right)$ show significant variations, especially in the outer disk $\left(r>70^{\prime \prime}\right)$. The strong north-south asymmetry in the vertical structure of NGC 4631 in the west side of the disk is caused by the diffuse stellar emission which is most pronounced in the north west regions above the disk. The diffuse stellar emission above the disk middle plane is thought to be the stellar debris detached from the stellar disk of NGC 4631. The similar color $(B-V \approx 0.45)$ for the diffuse emission and NGC 4631 might support the conjecture that they are stellar debris from the disk of NGC 4631.

Understanding the origin of the diffuse stellar emission in NGC 4631 is important for a better description of the vertical structure of NGC 4631. One thing worthy of note is that diffuse stellar emission is present all around the galaxy although it is more prominent in the north west regions above the disk. This means that the disk of NGC 4631 is disturbed globally. One plausible scenario for tidal debris as an origin for the diffuse stellar emission is that they are formed by close encounters with neighbor galaxies that have comparable masses. NGC 4656 which is $\sim 71 \mathrm{kpc}$ away to the south east is the most probable candidate for the close encounters which lead to tidal stripping of some disk materials because it is massive enough to detach gas and stars from the disk of NGC 4631. Combes (1978) showed that a parabolic encounter of NGC 4656 with NGC 4631 well explains the HI bridges and counterarm. Interactions with NGC 4627 seem to contribute to the diffuse stellar debris since other radio features of NGC 4631 observed by Weliachew et al. (1978) can be reproduced by taking NGC 4627 into account.

Disk heating due to molecular clouds or minor mergers could explain some thick disks. But this does not seem to be the case for NGC 4631, especially for the diffuse stellar debris above the disk plane. Disk heating by molecular clouds could result in constant scale height (van der Kruit \& Searle 1982) and minor mergers do not seem to be strong enough to detach the stellar disk of NGC 4631. However, tidal debris of the satellite galaxies that accreted to NGC 4631 may contribute to the diffuse stellar emission above the disk of NGC 4631.

\section{ACKNOWLEDGMENTS}

This work was supported for two years by Pusan National University Research Grant. 


\section{REFERENCES}

Abadi, M.G., Navarro, J. F., Steinmetz, M., \& Eke, V. R. 2003, Simulations of Galaxy Formation in a $\Lambda$ Cold Dark Matter Universe. I. Dynamical and Photometric Properties of a Simulated Disk Galaxy, ApJ, 591, 499

Ann, H. B., \& Park, J.-C. 2006, Warped Disks in Spiral Galaxies, New Astronomy, 11, 293

Ann, H. B. 2007, The Warped Disk of Integral-Sign Galaxy PGC 20348, JKAS, 40, 9

Bekki, K., \& Chiba, M. 2001, Formation of the Galactic Stellar Halo. I. Structure and Kinematics, ApJ, 558, 666

Bendo, G. J. et al. 2006, The Spectral Energy Distribution of Dust Emission in the Edge-On Spiral Galaxy NGC 4631 as Seen with Spitzer and the James Clerk Maxwell Telescope, ApJ, 652, 238

Brook, C. B., Kawata, D., Gibson, B. K., \& Freeman, K. C. 2004, The Emergence of the Thick Disk in a Cold Dark Matter Universe. ApJ, 612, 894

Burkert, A., Truran, J. W., \& Hensler, G. 1992, The Collapse of our Galaxy and the Formation of the Galactic Disk, ApJ, 391, 651

Burstein, D. 1979, Structure and Origin of S0 Galaxies. II - Disk-to-Bulge Ratios, ApJ, 234, 829

Carney, B. W., Latham, D. W., \& Laird, J. B. 1989, A Survey of Proper-Motion Stars. VIII - On the Galaxy's Third Population, AJ, 97, 423

Combes, F. 1978, A Model of Tidal Interactions within the NGC 4631 Group of Galaxies, A\&A, 65, 47

Crillon, R.; Monnet, G. 1969, Kinematic Study of Ionized Hydrogen in NGC 4631, A\&A, 2, 1

Dalcanton, J. J., \& Bernstein, R. A. 2002, A Structural and Dynamical Study of Late-Type, Edge-on Galaxies. II. Vertical Color Gradients and the Detection of Ubiquitous Thick Disks, AJ, 124, 1328

de Grijs, R., \& Peletier, R. F. 1997, The Shape of Galaxy Disks: How the Scale Height Increases with Galactocentric Distance, A\&A, 320, 21

Fabbiano, G., \& Trinchieri, G. 1987, X-Ray Observations of Spiral Galaxies. II - Images and Spectral Parameters of 13 Galaxies, ApJ, 315, 46

Fall, S. M., \& Efstathiou, G. 1980, Formation and Rotation of Disc Galaxies with Haloes, MNRAS, 193, 189

Freeman, K., \& Bland-Hawthorn, J. 2002, the New Galaxy: Signatures of Its Formation, ARA\&A, 40, 487

Fuhmann, K. 2004, Nearby Stars of the Galactic Disk and Halo. III, AN, 325, 3

Gilmore, G., \& Reid, N. 1983, New Light on Faint Stars. III - Galactic Structure towards the South Pole and the Galactic Thick Disc, MNRAS, 202, 1025
Gilmore, G. 1984, New Light on Faint Stars. VI - Structure and Evolution of the Galactic Spheroid, MNRAS, 207, 223

Gilmore, G., Wyse, R.F. G., \& Norris, J. E. 2002, Deciphering the Last Major Invasion of the Milky Way, ApJ, 574, L39

Giuricin, G., Marinoni, C., Ceriani, L., \& Pisani, A. 2000, Near by Optical Galaxies: Selection of the Sample and Identification of Group, ApJ, 543, 179

Helmi, A., Navarro, J. F., Nordstrom, B., Holmberg, J., Abadi, M. G., \& Steinmetz, M. 2006, Pieces of the Puzzle: Ancient Substructure in the Galactic Disc, MNRAS, 365, 1309

Hummel, E., \& Dettmar, R.-J. 1990, Radio Observations and Optical Photometry of the Edge-On Spiral Galaxy NGC 4631, A\&A, 236, 33

Hummel, E., Beck, R., \& Dahlem, M. 1991, The Magnetic Field Structure in the Radio Halos of NGC 891 and NGC 4631, A\&A, 248, 23

Ichikawa, S.-I., Okamura, S., Watanabe, M., Hamabe, M., Aoki, T., \& Kodaira, K. 1987, SPIRAL: Surface Photometry Interactive Reduction and Analysis Library, Ann. Tokyo Astron. Obs., 21, 285

Jones, B. J. T., \& Wyse, R. F. G. 1983, The Formation of Disc Galaxies, A\&A, 120, 165

Irwin, J. A. et al. 2011. The JCMT Nearby Galaxies Legacy Survey - V. The $\mathrm{CO}(\mathrm{J}=3-2)$ Distribution and Molecular Outflow in NGC, MNRAS, 410, 1423

Kazantzidis, S.; Bullock, J. S., Zentner, A. R., Kravtsov, A. V., \& Moustakas, L. A. 2008, Cold Dark Matter Substructure and Galactic Disks. I. Morphological Signatures of Hierarchical Satellite Accretion, ApJ, 688, 254

Kim, E. H. et al. 2011, in preparation

Kormendy, J. 1977, Brightness Distributions in Compact and Normal Galaxies. III - Decomposition of Observed Profiles into Spheroid and Disk Components, ApJ, 217, 406

Kroupa, P. 2002, Thickening of Galactic Discs through Clustered Star Formation, MNRAS, 330, 707

Lee, M. G., Freedman, W. L., \& Madore, Barry F. 1993. The Tip of the Red Giant Branch as a Distance Indicator for Resolved Galaxies, ApJ, 417, 553

Martin, C, \& Kern, B. 2001, Imaging and Spectroscopy of the Multi phase Halo of NGC 4631, ApJ, 555,258

Martin, N. F., Ibata, R. A., Bellazzini, M., Irwin, M. J., Lewis, G. F., \& Dehnen, W. 2004, A Dwarf Galaxy Remnant in Canis Major: the Fossil of an In-Plane Accretion on to the Milky Way, MNRAS, 348, 12

Navarro, J. F., Helmi, A., \& Freeman, K. C. 2004, The Extragalactic Origin of the Arcturus Group, ApJ, 601,43 
Neininger, N., \& Dumke, M. 1999, Intergalactic Cold Dust in the NGC 4631 Group, 1999, PNAS, 96, 5360

Pohlen, M., Dettmar, R.-J., Lutticke, R., \& Schwarzkopf, U. 2000, Three-Dimensional Modelling of Edge-On Disk Galaxies, A\&AS, 144, 405

Pohlen, M., Balcells, M., Lutticke, R., \& Dettmar, R. 2004, Thick Disks of Lenticular Galaxies. 3DPhotometric Thin/Thick Disk Decomposition of Eight Edge-On s0 Galaxies, A\&A, 422, 465

Quinn, P. J., Hernquist, L., \& Fullagar, D. P. 1993, Heating of Galactic Disks by Mergers, ApJ, 403, 74

Rand, R. J., Kulkarni, S. R., \& Hester, J. J. 1999, Warm Ionized Gas in the Edge-On Galaxies NGC 4565 and NGC 4631, ApJ, 396, 97

Rand, R. J. 1994, Atomic Hydrogen in the NGC 4631 Group of Galaxies, A\&A, 285, 833

Read, J. I., Lake, G., Agertz, O., \& Debattista, V. P. 2008, Thin, Thick and Dark Discs in $\Lambda$ CDM, MNRAS, 389, 1041

Robin, A. C., Haywood, M., Creze, M., Ojha, D. K., \& Bienayme, O, 1996, The Thick Disc of the Galaxy: Sequel of a Merging Event, A\&A, 305, 125

Roberts, M. S. 1968, Neutral Hydrogen Observations of the Binary Galaxy System NGC 4631/4656, ApJ, 151,117

Seth, A. C., Dalcanton, J. J., \& de Jong, R. S. 2005a, A Study of Edge-On Galaxies with the Hubble Space Telescope Advanced Camera for Surveys. I. Initial Results, AJ, 129, 1331

Seth, A. C., Dalcanton, J. J., \& de Jong, R. S. 2005b, A Study of Edge-On Galaxies with the Hubble Space Telescope Advanced Camera for Surveys. II. Vertical Distribution of the Resolved Stellar Population, AJ, 130,1574

Spitzer, L. Jr., \& Schwarzschild, M. 1951, The Possible Influence of Interstellar Clouds on Stellar Velocities, ApJ, 114, 385

Tsikoudi, V. 1979, Photometry and Structure of Lenticular Galaxies. I - NGC 3115, ApJ, 234, 842

van der Kruit, P. C., \& Searle, L. 1981a, Surface Photometry of Edge-On Spiral Galaxies. I - A Model for the Three-Dimensional Distribution of Light in Galactic Disks, A\&A, 95, 105

van der Kruit, P. C., \& Searle, L. 1981b, Surface Photometry of Edge-On Spiral Galaxies. II - the Distribution of Light and Colour in the Disk and Spheroid of NGC891, A\&A, 95, 116

van der Kruit, P. C., \& Searle, L. 1982, Surface Photometry of Edge-On Spiral Galaxies. III - Properties of the Three-Dimensional Distribution of Light and Mass in Disks of Spiral Galaxies, A\&A, 110, 61

Villalobos, A., \& Helmi, A. 2008, Simulations of Minor Mergers - I. General Properties of Thick Discs, MNRAS, 391, 1806
Vogler, A., \& Pietsch, W. 1996, ROSAT Observations of NGC 4631, A\&A, 311, 35

Velazquez, H., \& White, S. D. M. 1999, Sinking Satellites and the Heating of Galaxy Discs, MNRAS, 304, 254

Walker, I. R., Mihos, J. C., \& Hernquist, L. 1996, Quantifying the Fragility of Galactic Disks in Minor Mergers, ApJ, 460, 121

Wang, Q. D., Walterbos, R. A. M., Steakley, M. F.; Norman, Colin A., \& Braun, R. 1995, ROSAT Detection of Diffuse Hot Gas in the Edge-On Galaxy NGC 4631, ApJ, 439, 176

Wang, Q. D., Immler, S., Walterbos, R., Lauroesch, J. T., \& Breitschwerdt, D. 2001, Chandra Detection of a Hot Gaseous Corona around the Edge-On Galaxy NGC 4631, ApJ, 555, L99

Weliachew, L., Sancisi, R., \& Guelin, M. 1978, Aperture Synthesis Observations of the Neutral Hydrogen in the Galaxies NGC 4631/NGC 4656, A\&A, 65, 37

Yoachim, P., \& Dalcanton, J. L. 2006, Structural Parameters of Thin and Thick Disks in Edge-On Disk Galaxies, AJ, 131, 226

Yoachim, P., \& Dalcanton, J. L. 2008, Lick Indices in the Thin and Thick Disks of Edge-On Disk Galaxies, ApJ, 683, 707

\section{APPENDIX A. Decomposition of the Perpen- dicular Profiles of NGC 4631}

We decompose the 16 perpendicular profiles for each passband using the iterative profile decomposition technique introduced by Kormendy (1977). We approximate the luminosity profiles of the thin and thick disk by two exponential functions,

$$
I_{\text {thin }}(z)=I_{0, \text { thin }} \exp \left(-z / z_{\text {thin }}\right),
$$

and

$$
I_{\text {thick }}(z)=I_{0, \text { thick }} \exp \left(-z / z_{\text {thick }}\right) \text {, }
$$

where $I_{0, \text { thin }}$ and $I_{0, \text { thick }}$ are the central intensities of the thin disk and thick disk and $z_{\text {thin }}$ and $z_{\text {thick }}$ are their scale heights. In the iterative profile decomposition, we fitted each function to the observed profile at the radii where the thin disk or the thick disk dominate. At the beginning of the iteration the observed profile itself is fitted by the assumed function, but after initial solution for one component, the observed profile was subtracted by the extrapolated values of the fitted function before fitting. We presented the results of the profile decomposition in Fig. 8 and Fig. 9. 


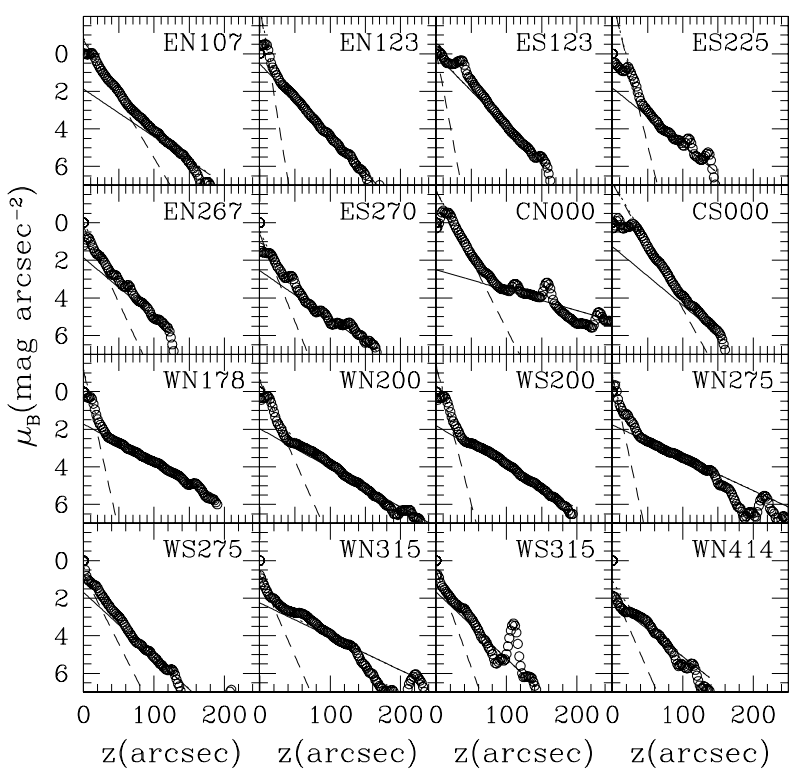

Fig. 8.- Profile decomposition of 16 perpendicular profiles in $B$-band. The dashed and solid lines represent the fitted functions of thin disk and thick disk, respectively. The dotted lines indicate the sum of the thin and thick disks but in most cases they are hardly seen because they are over plotted on the observed profiles. The labels in each panel shows the position and orientation of the profiles. For example, EN107 indicates the profile extracted at $r=107^{\prime \prime}$ in the east side of the disk, oriented to the north.

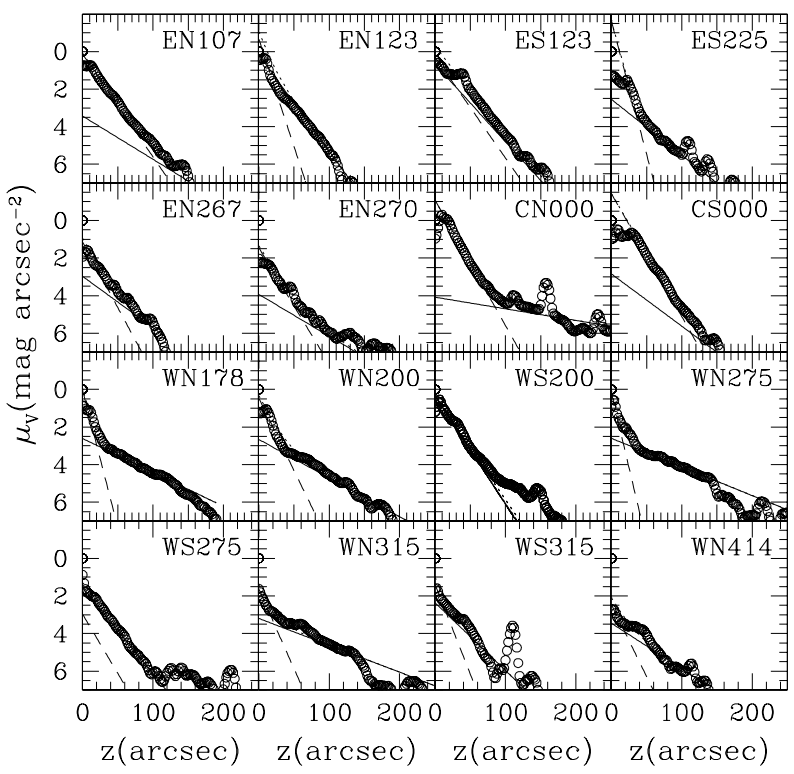

Fig. 9. - The profile decomposition of 16 perpendicular profiles in $V$-band. The meaning of lines is the same as in Fig. 8 . 